\title{
ANALYSIS AND IMPLEMENTATION OF BRAIN WAVES FEATURE EXTRACTION AND CLASSIFICATION TO CONTROL ROBOTIC HAND
}

\author{
Mohammed Z. Al-Faiz ${ }^{1}$, Ammar A. Al-Hamadani2 \\ ${ }^{1}$ Al-Farahidi University, Baghdad, Iraq \\ ${ }^{2}$ College of Information Engineering, Al-Nahrain University, Baghdad, Iraq \\ $\left\{\right.$ mzalfaiz12 ${ }^{1}$, eng.ammar.noori $\left.{ }^{2}\right\} @$ gmail.com \\ Received:8/10/2018, Accepted:30/10/2018
}

\begin{abstract}
In this paper, feature extraction methods such as time domain, frequency domain and spatial domain were investigated. Where Mean Absolute Value (MAV), Integrated Absolute Value (IAV), Zero Crossing (ZC), Root Mean Square (RMS), Waveform Length (WL) and Slope Sign Change (SSC) are the used time domain features. Autoregressive Feature (AR) is the frequency domain feature and the spatial domain feature is the Common Spatial Patterns (CSP). Channel selection algorithm was proposed for dimensionality reduction using Matlab code. Results of the above algorithm were compared with Matlab library of Principle Component Analysis (PCA). The extracted feature vectors were fed into Support Vector Machine with Radial Basis Function kernel (SVM-RBF) to train the classifier. The pair of algorithms (feature extraction plus dimensionality reduction) that owned the lowest classification error rate were used to control a Humanoid Robotic Hand (HRH) in offline mode. EEG dataset of two classes and three bipolar channels was used. Results showed that CSP features achieved the lowest classification error rate for both dimensionality reduction techniques with $\mathbf{2 . 1 4 \%}$. Results recommends to use (CSP plus channel selection algorithm) over (PCA plus PCA) since the former owned lowest classification processing time of 8.2s over 8.5s for the later.
\end{abstract}

Keywords: BCI, Humanoid robotic hand, Pattern recognition, Features extraction, Support vector machine, Common spatial patterns, Principal component analysis, Channel selection algorithm

\section{INTRODUCTION}

As an important practice in the brain computer interface (BCI) systems, specifically in humanoid robotic hand (HRH) applications, is to uncover the intention of human to move his hands. Such intention can be extracted from the electroencephalography (EEG) as a time series signal. The EEG signal can either be recorded (offline) to be used in further analysis or to directly control BCI system such as robotic or prosthetic hand (online). However, one of the limitation factors in the practical implementation of any BCI based EEG paradigm is the difficulty of discrimination (decoding the data). Such problem is due to the high dimension and the nonlinearity of EEG data which prevent the reliable discrimination and leads to lower classification accuracy [1]. Choosing appropriate feature extraction technique plays major roll towards successful computation of the correct class or hand gesture. Many techniques have been presented in the literature, of them, the paradigm presented in [2] to control prosthetic hand. Adaptive autoregressive (AAR) model has been used to extract features from EEG signal, linear discriminate analysis (LDA) has been used as classifier. Three sessions have been attempted and the best classification accuracies were 85.5, 88.75 and $90 \%$ respectively. In [3], novel method of online feature extraction and classification for BCI based EEG signal has been implemented. The method has been used to predict the left and right hand Motor Imagery (MI) by employing CSP feature and SVM classifier. Three classification results have been calculated for three subjects, the highest result was $92 \%$ for the third one. Researchers in [4] have used the invasive approach of Electrocorticography (ECoG) to control humanoid robot, the experiment was based on MI BCI. CSP feature 
extraction and LDA classifier have been used, the obtained classification accuracy was $95.4 \%$ after five runs. In another approach such as [5], BCI system with adaptive decoder and discrete wavelet transform (DWT) feature has been proposed. Up to 4th order Daubechies wavelet has been extracted. The feature vector has been constructed by taking the mean of the reconstructed signals of D3 and D4 tree levels. The features have been applied to SVM for classification with average decoding accuracy of $75 \%$ in online mode. In [6], dataset of BCI competition IV-IIa has been used to implement pattern recognition paradigm. Spatial feature (CSP) has been extracted from the data. Twin SVM classifiers have been used and optimal parameters of the SVM has been optimized using chaotic particle swarm optimization. The highest classification accuracy has been achieved with subject A08 at 97.21New Brain Machine Interface (BMI) system has been developed based on Imagined Body Kinematics (IBK) in [7]. This approach harnessed the shared control strategy in which the subject instructed to imagine the movement of virtual cursor to satisfy pre-programming target. Commands have been generated after hitting the target to control a robotic arm. The EEG signal has been filtered in the range of $0.16 \mathrm{and} 1 \mathrm{~Hz}$, such low frequency information regarded as features of the IBK. Linear regression has been used to decode the EEG signal. A custom metric called goodness-of-fit has been used to assess the classification accuracy and the success rate of the model was 70\%. In [8], time domain and frequency domain features have been proposed based on [9]. Six features have been placed in the feature vector and LDA classifier has been calculated with classification accuracy of $91.10 \%$. In this work, set of time domain, frequency domain [9] [10] and spatial domain (CSP) features [6] were extracted separately. Two dimensionality reduction algorithms based on [10] and [11] were implemented in two separate experiments. The first experiment was done using PCA algorithm with all the features (each feature was taken alone). The second experiment was done in the same way of the above but using the channel selection algorithm. SVM-RBF classifier was employed in both experiments to predict the class of motion from the EEG dataset. Performance comparison was made in terms of classification error rate. Where the classification error rate was calculated by using one of the feature methods with one of the dimensionality reduction algorithms at a time. The pair of algorithms (feature extractions plus dimensionality reduction) that have the lowest classification error rate were used to control robotic hand as an offline BCI paradigm.

\section{Proposed Method}

\section{A. Dataset}

Two-class MI EEG dataset was used to perform offline control of robotic hand. The dataset is provided by the Department of Medical Informatics, Institute for Biomedical Engineering, University of Technology Graz, Austria. Class 1 referred to left hand MI and class 2 referred to right hand MI. The dataset and its details are available at [12] where this is the only available reference and it's a trusted website. The signal was acquired from three bipolar channels placed on C3, Cz, C4 based on 10-20 international system. Data are sampled at $128 \mathrm{~Hz}$, bandpass filtered at $(0.5-30 \mathrm{~Hz})$.

\section{B. System Design}

All processing steps were done using Matlab codes programmed to fit the research needs. The general system architecture is shown in Fig.1 where the dataset are fed into the feature extraction then the classification module. After performing 


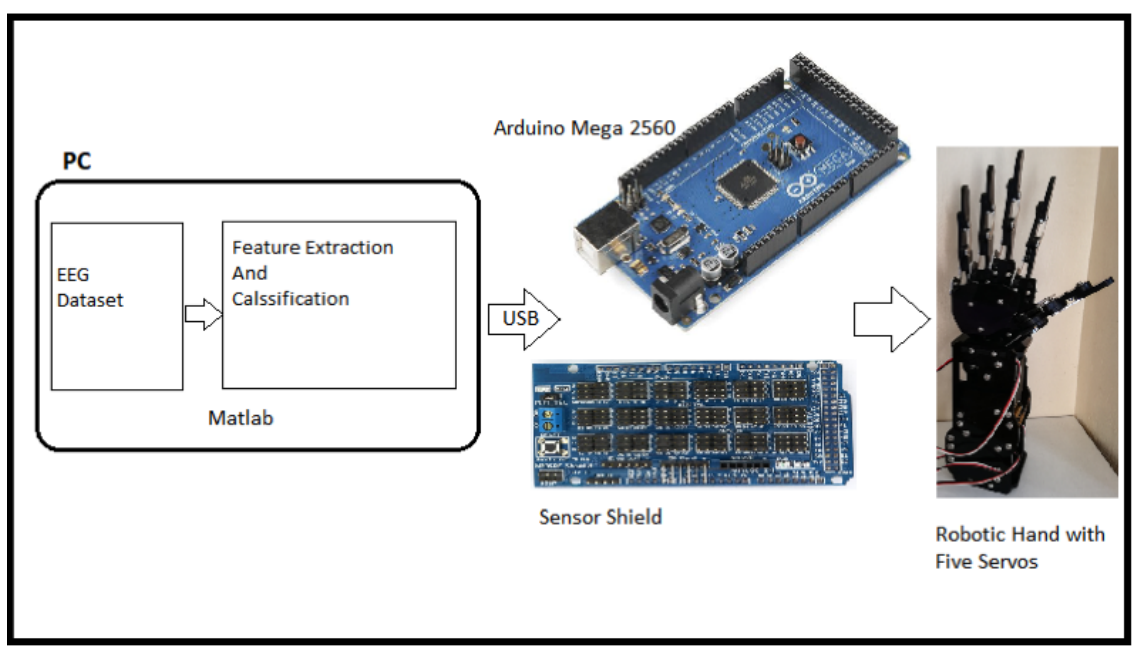

Figure 1: The essential locations of the FTs, they exist on the palm side skin of a little finger, ring finger, middle finger and index finger[2]

signal processing, the successfully classified signal was transferred via USB to arduino mega microcontroller as a command to control the HRH. Arduino mega sensor shield was used to connect Arduino with servos pins of the HRH.

The procedure of the proposed method can be explained as below:

1) Feature Extraction: To increase the classification performance, features of data was extracted and used as input to the classification algorithm instead of the raw EEG data. Time domain, frequency domain and spatial domain features was extracted and compared in terms of its impact on the rate of error for the classification algorithm. The experimented features are [9]:

- Mean Absolute Value (MAV)

$$
\frac{1}{N} \sum_{i=1}^{N}\left|X_{i}\right|
$$

- Integral Absolute Value (IAV)

$$
\sum_{i=1}^{N}\left|X_{i}\right|
$$

- Root Mean Square (RMS)

$$
\sqrt{\frac{1}{N} \sum_{i=1}^{N} X_{i}^{2}}
$$

- Wave Length (WL) 
- Zero Crossing (ZC)

$$
\sum_{i=1}^{N-1}\left|X_{i+1}-X_{i}\right|
$$

'Zero Crossing (ZC)

$$
\begin{gathered}
\sum_{n=1}^{N-1}\left[\operatorname{sgn}\left(X_{n} \times X_{n+1}\right) \cap\left|X_{n}-X_{n+1}\right| \geq \text { threshold }\right] \\
\operatorname{sgn}(x)=\left\{\begin{array}{c}
1, x \geq \text { threshold } \\
0, \text { Otherwise }
\end{array}\right.
\end{gathered}
$$

- Slope Sign Change (SSC)

$$
\begin{gathered}
\sum_{i=1}^{N-1}\left[\int\left[\left(X_{i}-X_{i-1}\right) \times\left(X_{i}-X_{i+1}\right)\right]\right] \\
f(x)=\left\{\begin{array}{c}
1, x \geq \text { threshold } \\
0, \text { Otherwise }
\end{array}\right.
\end{gathered}
$$

- Autoregressive (AR)

$$
-\sum_{i=1}^{p} a_{i} X_{i-1}+W_{n} p=4
$$

Equations (1-6) represents the time domain features while, Eq.7 is the only frequency domain feature that proposed to be used. In all the equations, $\mathrm{X}$ represent the EEG signal sample, $\mathrm{N}$ is the signal length. In Eq.7, $a_{i}$ represent the $\mathrm{i}^{\text {th }} \mathrm{AR}$ coefficient, $\mathrm{p}$ is the autoregressive order, and $W_{n}$ is the white noise, AR (4) is proposed to be used.

- Common Spatial Patterns (CSP) was the proposed spatial domain feature that calculated by taking the covariance matrix of the EEG channels and $\mathrm{T}$ is the samples per channel [6].

$$
\Sigma_{i}=\frac{X X^{\prime}}{\operatorname{trace}\left(X X^{\prime}\right)}
$$

Getting the average covariance matrix for both classes $\bar{\Sigma}_{1}$ and $\bar{\Sigma}_{2}$ to form the composite covariance matrix as in Eq.9.

$$
\Sigma=\bar{\Sigma}_{l}+\bar{\Sigma}_{r}
$$

Generalizing Eigen decomposition to get Eq.10

$$
\Sigma=a \lambda a^{\prime}
$$

Where, $a$ is the eigenvector and $\lambda$ its corresponding eigenvalue. Sorting $\lambda$ in descending order, $\lambda_{d}$, to calculate the transformation matrix as in Eq.11, 


$$
\varphi=\sqrt{a^{\prime} \lambda_{d}^{-1}}
$$

Taking the transformation of the covariance matrix for both class as in Eq.12. It can be seen that by adding the eigenvectors of both covariance matrices, identity matrix is formed as in Eq.13.

$$
\begin{gathered}
\varphi \bar{\Sigma}_{i} \varphi^{\prime}=S_{i}=U \theta_{i} U^{\prime} \\
\theta_{1}+\theta_{2}=I
\end{gathered}
$$

Calculating the projection matrix $\mathrm{W}$ to perform the CSP filtering on the EEG signal as in Eq.14 and Eq.15 respectively, $\mathrm{W}$ is also called the spatial filter and $\mathrm{Z}$ is the uncorrelated (filtered) EEG signal components:

$$
\begin{gathered}
W=\left(U^{\prime} \varphi\right)^{\prime} \\
Z=W X
\end{gathered}
$$

Finally, CSP features are extracted by taking the log-transformation of $\mathrm{Z}$ for each channel alone:

$$
C S P_{f e t}=\log \left(\frac{\operatorname{var}\left(Z_{c h}\right)}{\sum_{i=1}^{C} \operatorname{var}\left(Z_{i}\right)}\right)
$$

For features presented in Eqs. (1-7), Matlab library introduced in [9] was employed while, for CSP feature, custom Matlab code based on [5] was coded to process the EEG dataset.

2) Dimensionality Reduction: In order to reduce the time consumption of the classification module, two dimensionality reduction techniques were used. The first is the Principal Component Analysis (PCA), the second is channel selection algorithm to reduce the number of EEG channels. The best technique was selected based on its effect on classification error rate.

For PCA, Modified Matlab code of [9] was used to process the columns (channels) of the input feature matrix with principal component analysis. The PCA algorithm performs Eigen decomposition on the feature matrix to calculate the eigenvalues and its corresponding eigenvectors. The eigenvectors that corresponding to largest two eigenvalues was only selected as feature vectors. Custom Matlab code was written to perform the channel selection algorithm. The time domain feature matrix or frequency domain feature matrix or CSP features matrix each consist of ( $\mathrm{N}$ rows of data samples $\mathrm{x} 3$ columns of EEG channels) Each kind of feature was reduced separately to ( $\mathrm{N}$ x 2 columns of EEG channels) based on the two lowest variance channel entropies as in Eq.17.

In order to reduce the time consumption of the classification module, two dimensionality reduction techniques were used. The first is the Principal Component Analysis (PCA), the second is channel selection algorithm to reduce the number of EEG channels. The best technique was selected based on its effect on classification error rate. 
For PCA, Modified Matlab code of [9] was used to process the columns (channels) of the input feature matrix with principal component analysis. The PCA algorithm performs Eigen decomposition on the feature matrix to calculate the eigenvalues and its corresponding eigenvectors. The eigenvectors that corresponding to largest two eigenvalues was only selected as feature vectors. Custom Matlab code was written to perform the channel selection algorithm. The time domain feature matrix or frequency domain feature matrix or CSP features matrix each consist of ( $\mathrm{N}$ rows of data samples $\mathrm{x} 3$ columns of EEG channels). Each kind of feature was reduced separately to ( $\mathrm{N}$ x 2 columns of EEG channels) based on the two lowest variance channel entropies as in Eq.17.

$$
I_{c h}=-\sum_{m=1}^{n} \operatorname{var}\left(C S P_{c h}^{m}\right) \cdot \ln \left(C S P_{c h}^{m}\right)
$$

Where, is the channel's variance entropy with $n=2$ representing the number of MI classes. The detailed design of channel selection algorithm is explained in the flowchart of Fig.2.

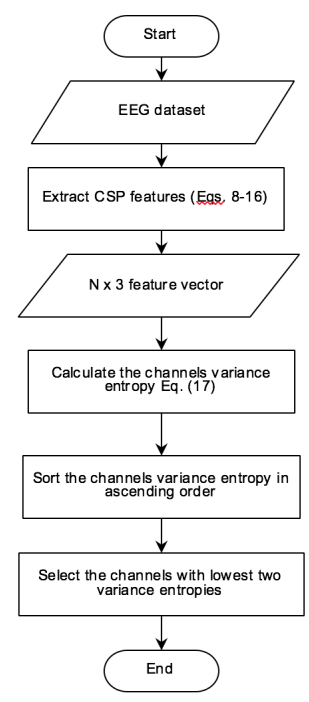

Figure 2: Flowchart of the proposed variance entropy channels selection algorithm

3) Classification: Support Vector Machine (SVM) algorithm was optimized for the purpose of EEG classification into left and right motor imagery (MI). The left hand MI was used to close the robotic hand's fist while the right hand MI was used to open it. The Matlab function fitcsvm was used to train the algorithm where $\mathrm{x}$-train part of the dataset was used for the training and $\mathrm{x}$-test part for testing. Radial bases kernel function (RBF) was chosen to the purpose of classification since the MI dataset has non-linearly separable property. RBF Kernel function is regarded as extension to SVM algorithm. RBF replaces each dot product in the algorithm with the function presented in Eq. (18) [13]. This function projects the input feature space into higher dimensional space so that the margin between the feature samples is maximized. Such approach 
enhances the discrimination performance and enlarge the separating hyperplane between the different classes.

$$
K\left(x_{i}, x_{j}\right)=\exp \left(-\left\|x_{i}-x_{j}\right\|^{2}\right)
$$

Where, is the transpose of in of the input feature matrix.

4) Controller and Robotic Hand: Arduino Mega 2560 was used as microprocessor module to control the robotic hand. The module was connected to Matlab via serial port (USB) in order to take the commands signals. The Matlab is communicating with the Arduino module with baudrate of 9600 signals per second. The robotic hand used is right hand, already assembled, made of black acrylic 5.0, 780g of weight. Height and width specifications are shown in Fig.3.
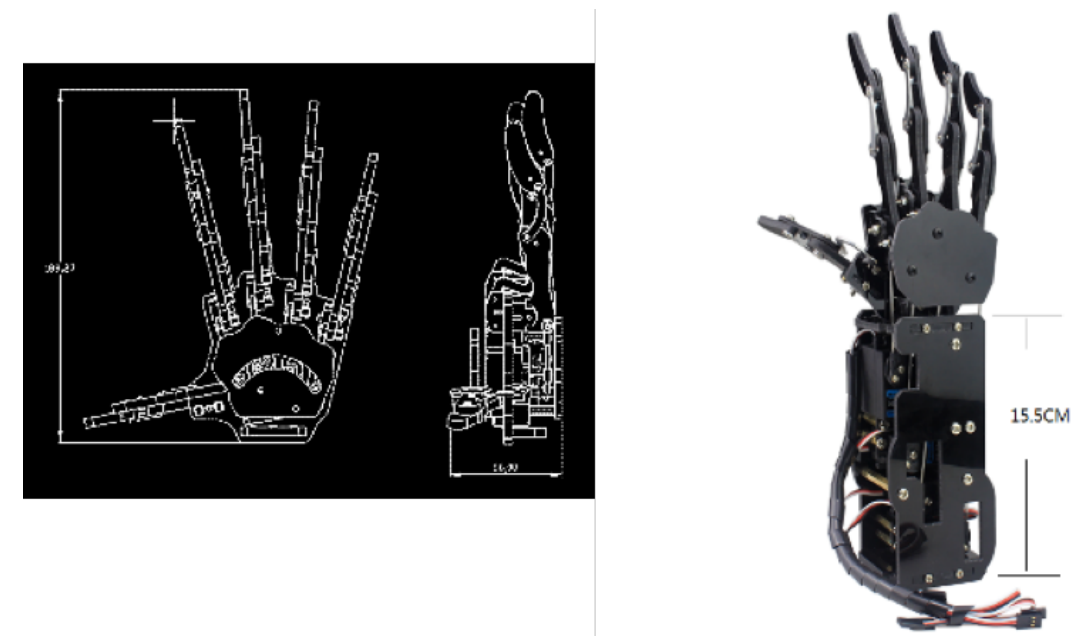

Figure 3: Dimensions of the robotic hand

The fingers were driven by five servos (Model: MagicQ DS15KG) with $68.4 \mathrm{v}$ working voltage and $>100 \mathrm{~mA}$ current. Rotation speed is 0.18 sec60 degree and the stall torque is $17 \mathrm{kgcm} 8.4 \mathrm{v}$. The hand's servos were connected to the PWM pins of the Arduino microcontroller to take the commands signals from Matlab via USB. The performance of the servos showed very slight effect of slow speed due to the small difference between its operating voltage and the 5 volts output voltage of PWM. The proposed system setup is presented in Fig.4.

After the successful prediction of the input feature to its classes, Matlab code was designed to send specific character "char" assigned for each class motion to the Arduino. $\mathrm{C}++$ code is designed and uploaded to the Arduino that translates each specific character sent by Matlab into specific hand's fingers movements. The fingers movements was achieved by rotating the corresponding servo to a predefined degree.

\section{RESUlTS AND DISCUSSION}

The research objectives were to perform set of feature extractions and dimensionality reduction algorithms on EEG dataset. The performance of each algorithm was assessed in terms of classification error rate and its corresponding classification accuracy. The pair of algorithms (feature extraction and dimensionality reduction) that owned the higher performance were 


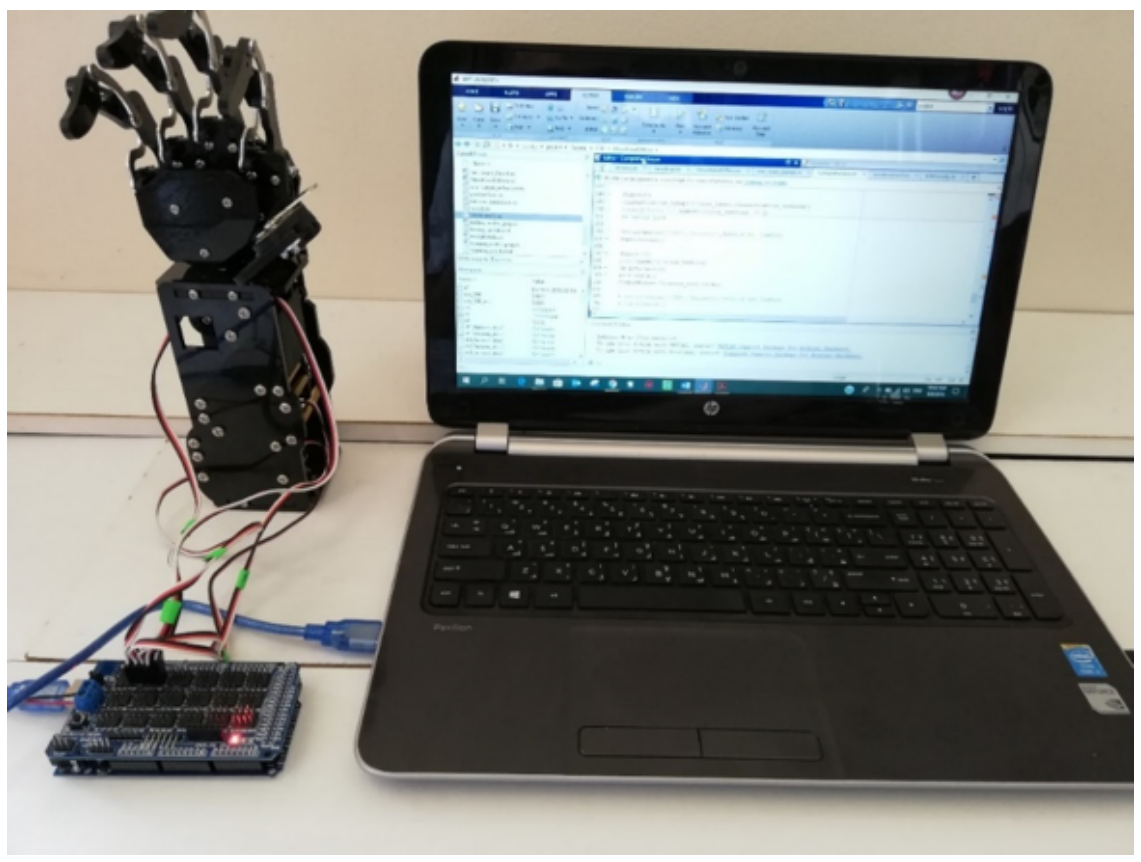

Figure 4: System setup of the offline controlled robotic hand using EEG signal dataset

used to run an offline BCI paradigm that controls robotic hand in two motions (i.e. Open and close the hand's fist). Table I shows comparison between the results of using the proposed features with both channel selection and PCA.

TABLE I

Classification Error Rate of Different Features Using Two DifFerent Dimensionality Reduction Techniques, VAlue Between Parentheses In No.8 Represents the Classification Processing Time.

\begin{tabular}{|l|l|l|l|}
\hline No. & Feature & Classification Error rate & Classification Error rate \\
\hline & & Channel selection & PCA \\
\hline 1 & MAV & $30.2 \%$ & $22.82 \%$ \\
\hline 2 & IAV & $30.2 \%$ & $22.82 \%$ \\
\hline 3 & RMS & $26.17 \%$ & $23.49 \%$ \\
\hline 4 & WL & $30.2 \%$ & $26.17 \%$ \\
\hline 5 & ZC & $54.36 \%$ & $51 \%$ \\
\hline 6 & SSC & $46.98 \%$ & $29.19 \%$ \\
\hline 7 & AR & $45.63 \%$ & $45.97 \%$ \\
\hline 8 & CSP & $2.14 \%(8.2 \mathrm{~s})$ & $2.14 \%(8.5 \mathrm{~s})$ \\
\hline
\end{tabular}

The results presented in Table I shows that PCA outperforms the channel selection algorithm in the time domain features (no. 16) while, the channel selection algorithm outperforms PCA in the frequency domain feature as in (no. 7). CSP feature experience the same performance for both techniques except that the classification processing time using channel selection algorithm is $8.2 \mathrm{~s}$ which considered lower than $8.5 \mathrm{~s}$ for PCA. To measure the processing time, tic toc Matlab function was used, note that this function was tested on PC with Intel Core i74500U CPU $1.80 \mathrm{GHz}$ and $8 \mathrm{~GB}$ of RAM. The tic function was placed just before performing the classification part and the toc function was placed after the classification part in the main Matlab code. Thus, the function measured the time elapsed between the start and end of the classification process. 
Figure 5 shows the classification error rate along with confusion matrix. The highest classification error rate was achieved using the channel selection algorithm with $\mathrm{ZC}$ feature at $54.36 \%$ as in Fig.5a. The lowest error rate was obtained using CSP feature with both dimensionality reduction algorithms at 2.14\% as in Fig.5b.
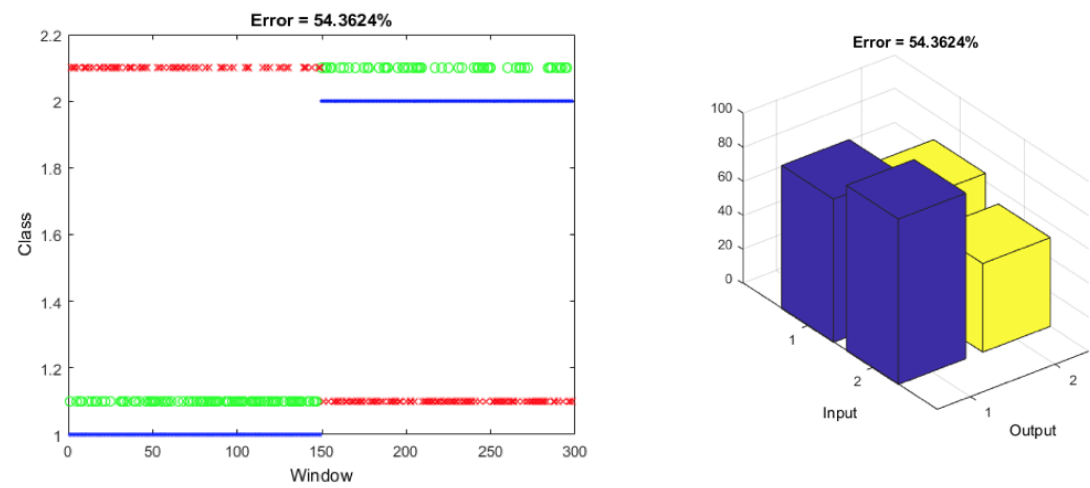

(a) ZC feature
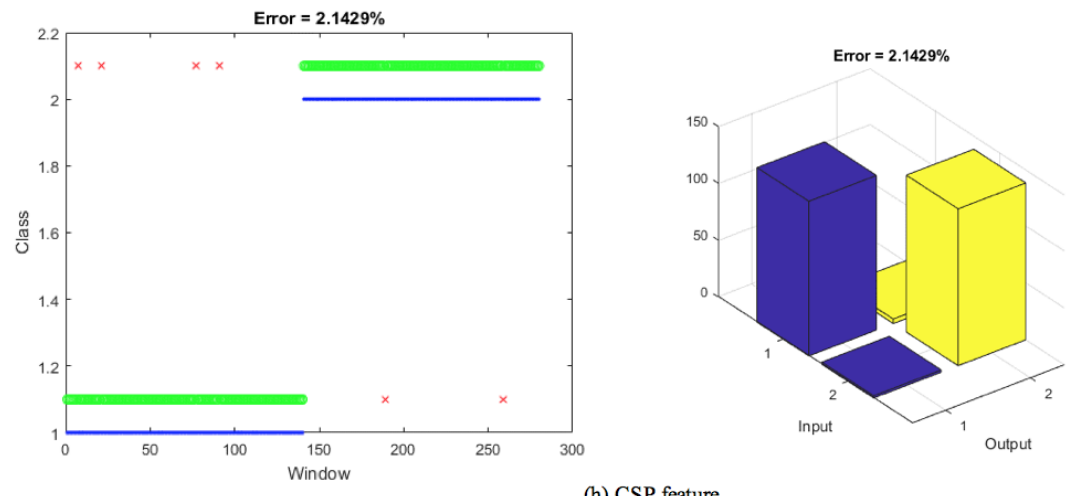

(h) CSP feature

Figure 5: Highest and lowest classification error rates per feature extraction method

Figure 5 (left side) shows the correct and incorrect classification for each motion class of SVM classifier. The Yaxis is the motion class number, while the Xaxis is the window sample number. Each window sample was tested for both motion classes and classification results were recorded. Correct classification is shown in green dot while an error is drawn as red $\mathrm{x}$, the blue line represents the true class. Fig.5 (right side) shows the confusion matrix of classifying two class motions where Zaxis is the number of samples, Xaxis is the input ( actual or target) class and Yaxis is the output ( predicted) class. The testing results showed that using CSP features to train SVM has the lowest classification error rate among all methods and thus has the highest classification accuracy of $97.86 \%$. The classification accuracies for the proposed feature extraction methods are presented in Fig.6.

Figure 6 shows that CSP feature extraction algorithm is quite successful in classifying EEG data. Implementation of CSP plus variance entropy channel selection and SVM paradigm to control the HRH is shown in Fig.7. 


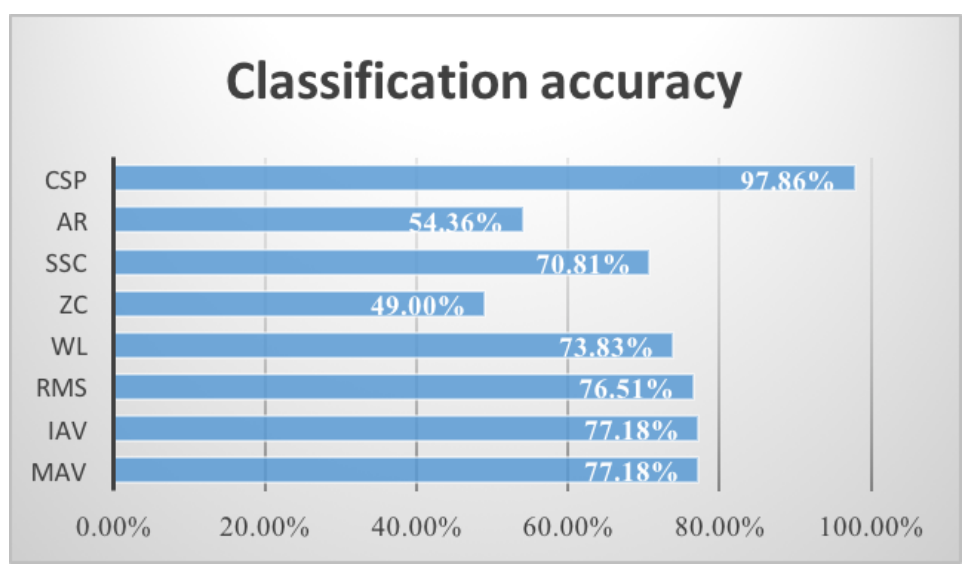

Figure 6: Classification accuracies result from using eight feature extraction methods with SVM classification algorithm

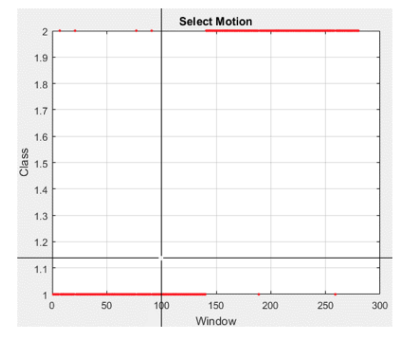

(a) Select class 1

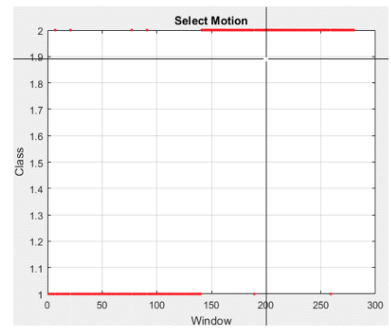

(c) Select tclass 2

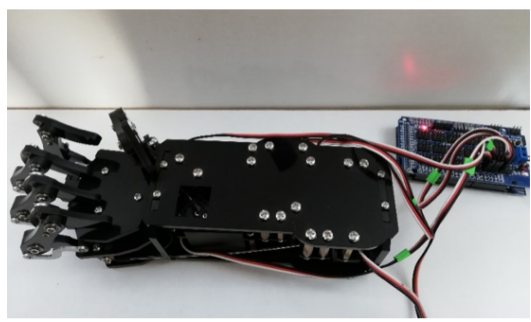

(b) Motion 1 (hand c lose)

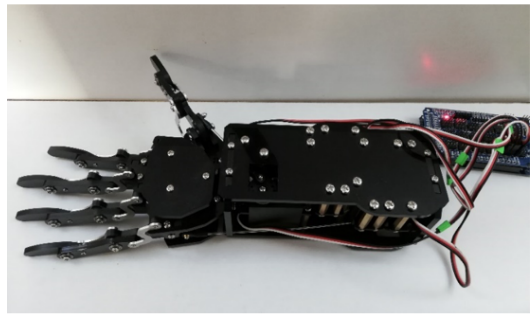

(d) Motion 2 (hand open)

Figure 7: Offline implementation of the proposed (CSP + variance entropy channel selection + SVM) paradigm to control the HRH

Results of the successfully classified testing data (x-test) are presented in Fig.7 (a-c). Matlab function ginput was used to represent the data as graphical input where, the desired motion (class) is select from mouse curser. Selecting one sample from class 1 in (a) results in closing the hand as in (b) and selecting one sample from class 2 in (c) results in opening the hand as in (d).

\section{Conclusion}

The objectives of this work are set of procedures to implement an offline BCI that controls humanoid robotic hand. The first objective is to perform set of time domain, frequency domain and spatial domain feature extraction algorithms 
separately. The second objective is to apply two dimensionality reduction technique in two separate experiments for each kind of feature. The first experiment is done using PCA and the second one is done using variance entropy channel selection on each of the extracted features. The third objective is to perform performance comparison in terms of classification error rate. The pair of algorithms (feature extractions plus dimensionality reduction) that have the lowest classification error rate is used to control the HRH. Using variance entropy channel selection algorithm shows the following classification error rate: $30.2 \%, 30.2 \%, 30.2 \%, 46.98 \%, 26.17 \%$ and $54.36 \%$ for MAV, WL, IAV, SSC, RMS and ZC respectively as time domain features. While $45.63 \%$ for AR as frequency domain feature and $2.14 \%$ for CSP as spatial domain feature. Using PCA algorithm shows the following classification error rate: 22.8\%, 26.17\%, 22.8\%, 29.19\%, 23.49\% and 51\% for MAV, WL, IA, SSC, RMS and ZC respectively as time domain features. While $45.97 \%$ for AR as frequency domain feature and $2.14 \%$ for CSP as spatial domain feature. Therefore, CSP features is quite successful in the classification of EEG signal and owns the highest classification accuracy among them all of $97.86 \%$. The above results shows two cases: the first case is that time domain features achieves higher accuracy when used with PCA, frequency domain feature achieves higher accuracy when used with channel selection algorithm. The spatial domain CSP features have the same performance with both dimensionality reduction techniques. The second case shows that using CSP with variance entropy channel selection has lowest classification processing time of $8.2 \mathrm{~s}$ as compared to $8.5 \mathrm{~s}$ when used with PCA. Thus the pair (CSP plus variance entropy channel selection) was used to train the SVM classifier and to control the HRH.

\section{REFERENCES}

[1] F. Lotte, " A Tutorial on EEG Signal processing Techniques for Mental state Recognition in Brain Computer Interfaces," Guide to Brain Computer Music Interfacing, pp. 133 to 161, 2014.

[2] C. Guger, W. Harkam, C. Hertnaes, and G. Pfurtscheller, " Prosthetic control by an EEG-based brain-computer interface (BCI)," In Proc. aaate 5th european conference for the advancement of assistive technology, pp. 36, 1999.

[3] H. Sun, Y. Xiang, Y. Sun, H. Zhu, and J. Zeng, "On-line EEG classification for brain computer interface based on CSP and SVM," 2010 3rd International Congress on Image and Signal Processing, vol. 9, pp. 4105 to 4108, 2010.

[4] C. Kapeller, P. Gergondet, K. Kamada, H. Ogawa, F. Takeuchi, R. Ortner, R. Pruckl, A. Kheddar, J. Scharinger, C. Guger, "Online control of a humanoid robot through hand movement imagination using CSP and ECoG based features," Conf Proc IEEE Eng Med Biol Soc. 2015, pp. 17658, Aug. 2015.

[5] S. Bhattacharyya, S. Shimoda, and M. Hayashibe, "A Synergetic Brain Machine Interfacing Paradigm for Multi DOF Robot Control," IEEE Transactions on Systems, Man, and Cybernetics: Systems, vol. 46, no. 7, pp. 957 to 968, 2016.

[6] L. Duan, Z. Hongxin, M. S. Khan, and M. Fang, "Recognition of motor imagery tasks for BCI using CSP and chaotic PSO twin SVM," The Journal of China Universities of Posts and Telecommunications, vol. 24, no. 3, pp. 83 to 90, 2017.

[7] J. Kilmarx, R. Abiri, S. Borhani, Y. Jiang, and X. Zhao, "Sequence based manipulation of robotic arm control in brain machine interface," International Journal of Intelligent Robotics and Applications, vol. 2, no. 2, pp. 149 to 160, Mar. 2018.

[8] M. Z. Al-Faiz and Z. A. Aljumaily, "Humanoid Robotic Hand (HRH) Based on EMG signal for Amputees Persons," International Journal of Emerging Trends in Engineering Research, vol. 6, no. 4, pp. 19 26, Jun. 2018.

[9] A. Phinyomark, C. Limsakul, and P. Phukpattaranont, "A novel feature extraction for robust EMG pattern recognition," JOURNAL OF COMPUTING, vol. 1, no. 1, pp. $7180,2009$.

[10] A. Chan and G. Green, "Myoelectric Control Development Toolbox," CMBES, vol. 30, no. 1, Nov. 2017.

[11] H. Sun, Y. Zhang, B. J. Gluckman, X. Zhong, and X. Zhang, "Optimal-channel Selection Algorithms in Mental Tasks based Brain-computer Interface," Proceedings of the 2018 8th International Conference on Bioscience, Biochemistry and Bioinformatics - ICBBB $2018,2018$.

[12] "BCI Competition II," BCI Competition IV. [Online]. Available: http://www.bbci.de/competition/ii/. [Accessed: 07-Oct-2018].

[13] "Nonlinear Transformation with Kernels," Mathworks documentation. 